\title{
LOS FACTORES SOCIOLABORALES Y SU INFLUENCIA EN EL NIVEL DE ESTRÉS DEL PERSONAL DE ENFERMERÍA DEL HOSPITAL III DANIEL ALCIDES CARRIÓN ESSALUD DE TACNA, PERÍODO 2009.
}

\author{
Autora: Guillermina Ethel Pacheco Alponte' \\ Universidad Privada de Tacna \\ E-mail: gpachecoa62@yahoo.com
}

\begin{abstract}
RESUMEN
El trabajo corresponde a una investigación básica, de naturaleza descriptiva correlacional debido a que está basado en especificar propiedades, características y determinar la relación de las variables de estudio: factores sociolaborales y nivel de estrés. La información se obtuvo con la aplicación del cuestionario aplicado por Maslach Burnout Inventory (MBI).La población de estudio está representada por el personal de enfermería deI Hospital III Daniel Alcides Carrión de Tacna (ESSALUD).

EI objetivo fue determinar la influencia de los factores sociolaborales en el estrés (agotamiento emocional, despersonalización y realización personal) presentado por el personal de enfermería del Hospital III Daniel Alcides Carrión ESSALUD de Tacná, período 2009.

Los resultados más importantes son: el personal de enfermería del Hospital III Daniel Alcides Carrión ESSALUD de Tacna, que pertenece al estado civil soltero; al rango de edad de adulto joven y que no tienen hijos, tienen mayores niveles de estrés. Asimismo se comprobó que el personal que tiene el menor tiempo de servicio, y que tienen horario de oficina tienen mayores niveles de estrés; determinándose además que el personal que labora en los servicios críticos de Emergencia, Unidad de Cuidados Intensivos (UCI) y Centro Quirúrgico, tiene mayores niveles de estrés; y que el nivel de estrés según agotamiento emocional, despersonalización es alto, existiendo una baja realización personal en la población de estudio.
\end{abstract}

\section{PALABRAS CLAVE:}

Nivel de estrés, Factores Sociolaborales, Sindrome de Burnout, Insatisfacción Laboral.

\begin{abstract}
This research work is a basic, descriptive, correlational work based on specifying properties, characteristics and determination of the relationship between study variables namely : social and job factors and level of stress . Information was obtained by applying the Maslach Burnout Inventory (MBI).Study population was formed by nurses of the Hospital III Daniel Alcides Carrión de Tacna (ESSALUD).
\end{abstract}

Our objective was to determine the influence of social and job factor son stress (emotional burning out, depersonalization and personal realization), as present in nurses of the Hospital III Daniel Alcides Carrion ESSALUD de Tacna, period 2009.

Most important results were : nurses in the Hospital III Daniel Alcides Carrión ESSALUD Tacna, who are single, Young adults and have no children show higher levels of stress. We also demonstrated that nurses with a lower time of work and have office schedules show higher levels of stress. The level of stress in personnel working in critical emergency services and intensive care units (ICUs) as well as surgical centers have higher levels of stress. The stress level graded by emotional burning out and depersonalization is high. There is a low level of selfrealization in studied population.

\section{KEYWORDS:}

Stress levels, working and social factors, burnout syndrome, job insatisfaction.

\footnotetext{
1 MG. ENENFERMERIA
} 


\section{INTRODUCCIÓN}

Para la atención de pacientes en los servicios del Hospital III Daniel Alcides Carrión ESSALUD de Tacna, se cuenta con un total de 108 enfermeras y demás profesionales de la salud, observándose que los mencionados profesionales, debido a factores sociolaborales, vienen presentado determinados niveles de estrés; ya sea por los años de servicio, el tipo de servicio en que labora, sexo, edad, número de hijos y horario de trabajo. Además, es probable que el personal de enfermería esté presentando estrés, el cual estaria en función del agotamiento emocional; o sea, respuestas emocionales, ya sea por sentimientos de preocupación, tensión, ansiedad, fatiga y/o agotamiento. Asimismo, otra fase del estrés que podría estar presentándose, es la despersonalización; ya que se observan cambios en la conducta y en la actitud del trabajador, lo que estaria ocasionando un trato impersonal y poco cordial hacia los pacientes. Es posible también que la insatisfacción; ya sea por factores salariales o sobre beneficios profesionales, podrian estar determinando su realización personal.

Siendo la pregunta de investigación ¿Cómo influyen los factores sociolaborales en el estrés (agotamiento emocional, despersonalización y realización personal) presentado por el personal de enfermeria del Hospital III Daniel Alcides Carrión ESSALUD de Tacna, período 2009?; se plantea la siguiente hipótesis: Los factores sociolaborales influyen en los altos niveles de estrés (agotamiento emocional, personalización y realización personal) presentado por el personal de enfermería del Hospital III Daniel Alcides Carrión.

La investigación constituye un aporte para el área de la salud; sobre todo, para garantizar un mejor servicio a la población asegurada de Tacna.

\section{MATERIALESY MÉTODOS}

El presente estudio adoptó un diseño no experimental, de tipo descriptivo correlacional. Se considera no experimental; por cuanto no se manipula la variable independiente factores sociolaborales, es decir, se observa tal y como se da en su contexto natural para luego analizarla. Es de tipo descriptivo correlacional; porque se busca medir las variables y la relación existente entre ambas.

La población de estudio está representada por el personal de enfermeria del Hospital III Daniel Alcides Carrión de Tacna (ESSALUD) ( 99 enfermeras), aplicándose para la recolección de datos el cuestionario de Maslach Burnout Inventory (MBI); bajo la técnica de la encuesta. El instrumento de medición ha sido tomado del estudio de investigación: Sindrome de Burnout en el personal de enfermería del servicio de Terapia Polivalente Hospital Saturnino Lora, el cual ha sido adaptado según objetivos de la investigadora, y sometido a la prueba de confiabilidad y validez.

Los resultados alcanzados fueron los siguientes:

\section{RESULTADOS \\ CUESTIONARIO INFLUENCIA DE LOS FACTORES SOCIOLABORALES EN EL NIVEL DE ESTRÉS}

\section{Gráfico $\mathrm{N}^{\circ} 01$ \\ Distribución de frecuencia de características sociodemográficas.}

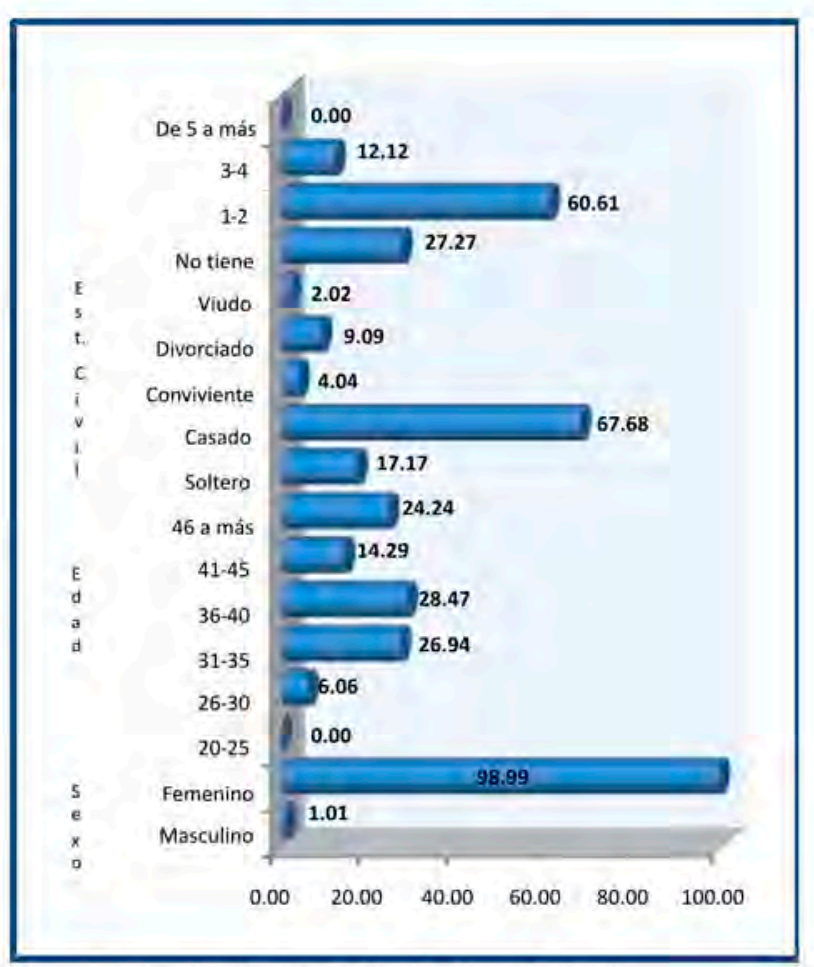

Fuente: Cuestionario sobre Estrés Laboral. Elaboración: Propia

En el gráfico se observa que el $98.09 \%$ pertenece al sexo femenino y el $1.01 \%$ al sexo masculino. Según edad el mayor porcentaje, el $28.47 \%$, comprende el rango de 36 a 40 años. El $67.68 \%$ es casado. Asimismo, se puede conocer que el $60,61 \%$ del personal de enfermeria tiene entre 1 a 2 hijos. 
Gráfico $\mathrm{N}^{\circ} 02$

Distribución de frecuencia de características laborales.

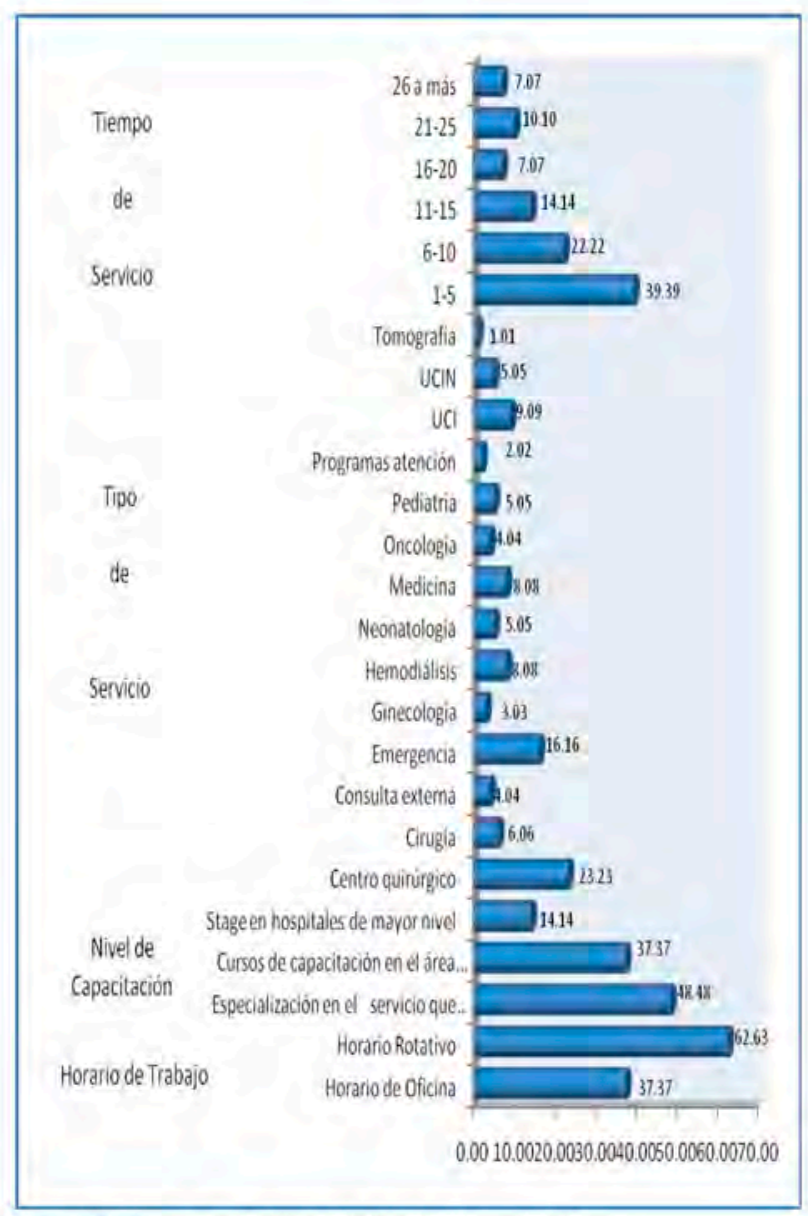

Fuente: Cuestionario sobre Estrés Laborai. Elaboración: Propia

En mayores porcentajes se tiene que el $39,39 \%$ del personal encuestado manifestó que pertenece al rango de I a 5 años de servicio; en cuanto al tipo de servicio, el $23,23 \%$ labora en el Centro quirúrgico; y en lo concerniente al nivel de capacitación, el $48,00 \%$ asevera que tiene especialización en el servicio que labora.
Gráfico $\mathrm{N}^{\circ} 03$

Distribución de frecuencia de los factores sociodemográficos según presencia de agotamiento emocional en el personal de Enfermería del Hospital III Daniel Alcides Carrión Essalud Tacna.

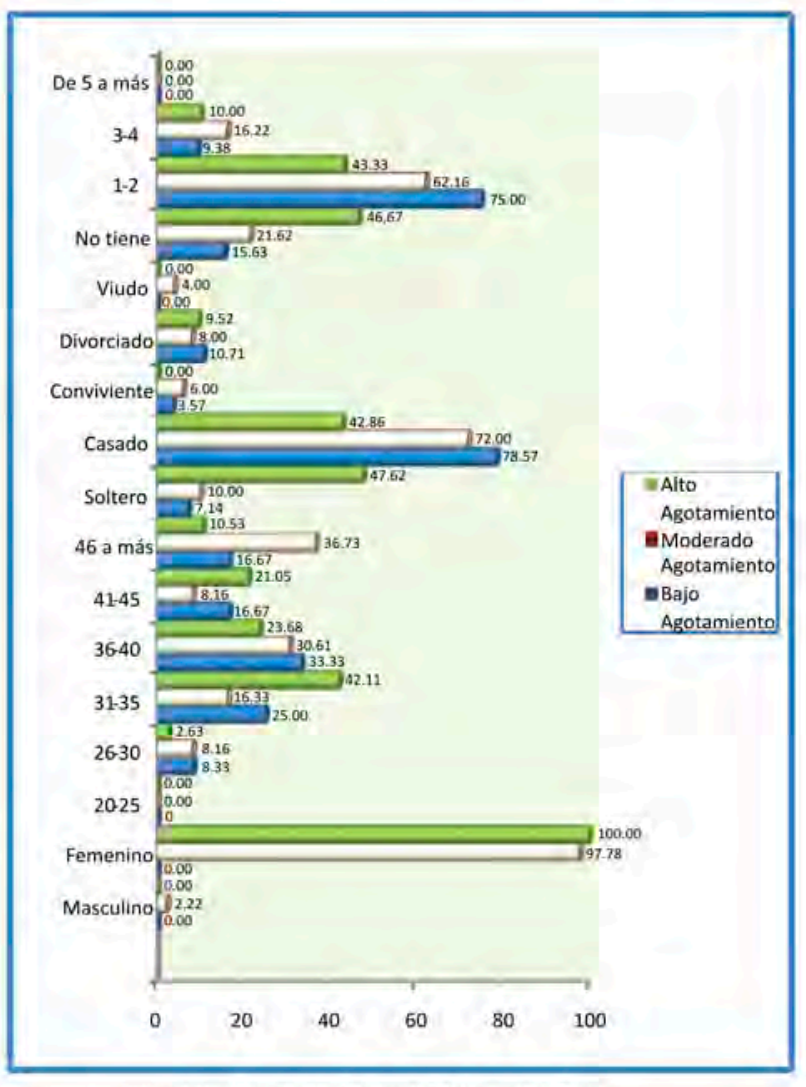

Fuente: Cuestionario sobre Estres Laboral. Elaboración: Propia

El factor asociado a la edad (p: 0,633). El adulto joven (31 a 35 años) presenta mayores niveles de estrés. Asimismo, se observa que a mayor edad, la frecuencia de alto agotamiento disminuye y esta asociación es altamente significativa.

En cuanto al factor asociado al estado civil (p: 0.082). Destaca que los solteros presentan los mayores niveles de estrés.

El factor asociado al número de hijos (p; 0,822). Las personas que no tienen hijos, tienen los mayores niveles de estrés. Pudiéndose deducir que quienes tienen hijos parecen más resistentes ante el Burnout, debido a que la implicación del sujeto con la familia le brinda mayor capacidad para afrontar los problemas y conflictos emocionales, pero también le permite ser más realistas con la ayuda del apoyo familiar. 


\section{Gráfico $\mathrm{N}^{\circ} 04$ \\ Distribución de frecuencia de los factores \\ sociodemográficos según presencia de despersonalización en el personal de Enfermería del Hospital III Daniel Alcides Carrión Essalud Tacna.}

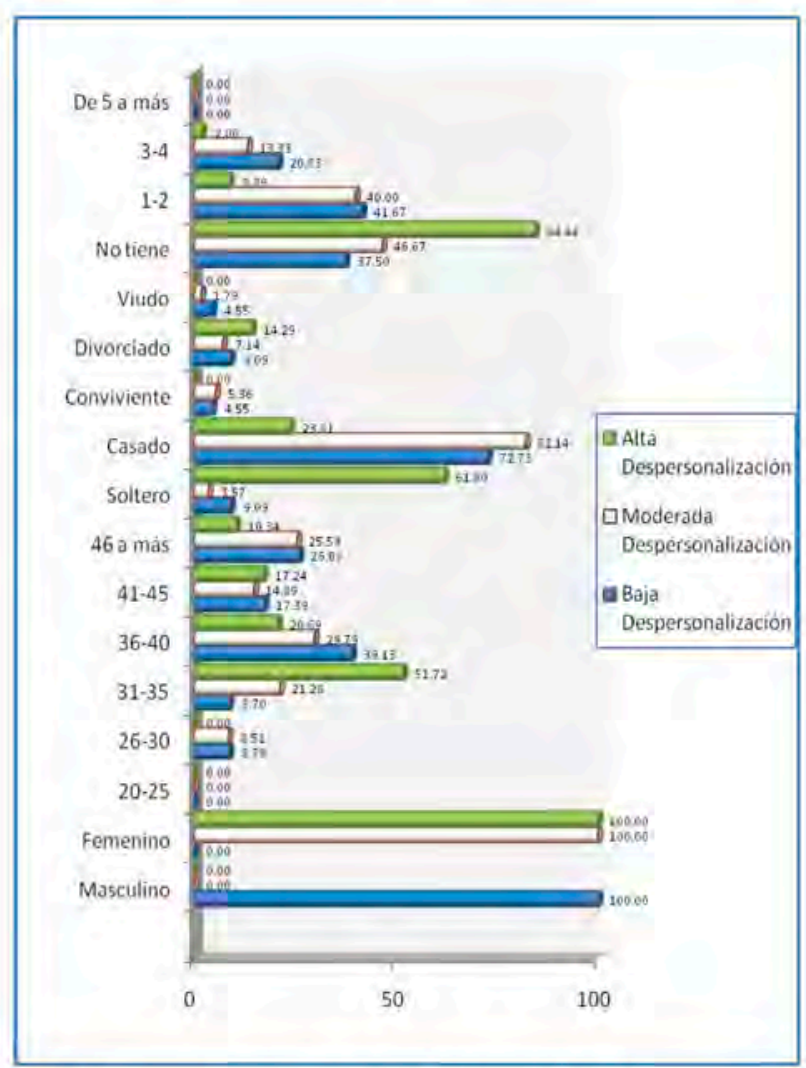

Fuente: Cuestionario sobre Estrés Laboral. Elaboración: Propia

El factor asociado a la edad (p: 0,032). Se encontró que quienes presentan el rango de edad de adulto joven (31-35 años) presentan mayor despersonalización. Asimismo, se observa que a mayor edad, la frecuencia de alta despersonalización disminuye y esta asociación es altamente significativa.

En cuanto al factor asociado al estado civil (p: 0.309). Se observa que los solteros presentan mayor despersonalización. Observándose, además, que esto disminuye de acuerdo al estado civil casado y estabilidad en pareja.

El factor asociado al número de hijos (p: 0,334). Implica que las personas que no tienen hijos, tienen mayor despersonalización; y esta relación es altamente significativa.

\section{Gráfico $\mathrm{N}^{\circ} 05$ \\ Distribución de frecuencia de los factores sociodemográficos según realización personal en el personal de Enfermería del Hospital III Daniel Alcides Carrión Essalud Tacna.}

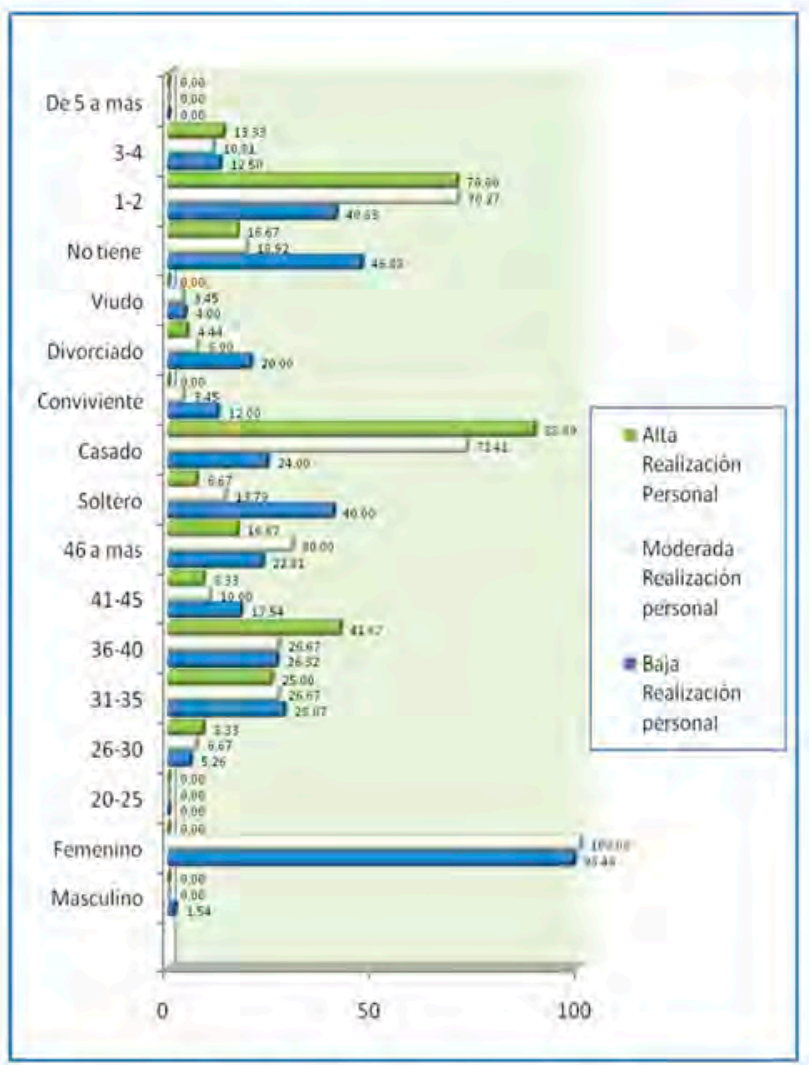

Fuente: Cuestionario sobre Estrés Laboral. Elaboración: Propia

El factor asociado a la edad es (p; 0,916). Aquellos que pertenecen al rango de edad de adulto joven (31-35 años) presentan baja realización personal. Asimismo, se observa que a mayores años de servicio, la frecuencia de alta realización personal disminuye; y esta asociación es altamente significativa.

El factor asociado al estado civil (p: 0.234). Se observa que los solteros presentan una menor realización personal. Además, se aprecia que este disminuye de acuerdo al estado civil casado y estabilidad en pareja.

El factor asociado al número de hijos (p: 0,857$)$. Implica que las personas que no tienen hijos, tienen menor realización personal; y esta relación es altamente significativa. 
Gráfico $\mathrm{N}^{\circ} 06$

Distribución de frecuencia de los factores laborales según agotamiento emocional en el personal de Enfermería del Hospital III Daniel Alcides Carrión Essalud Tacna.

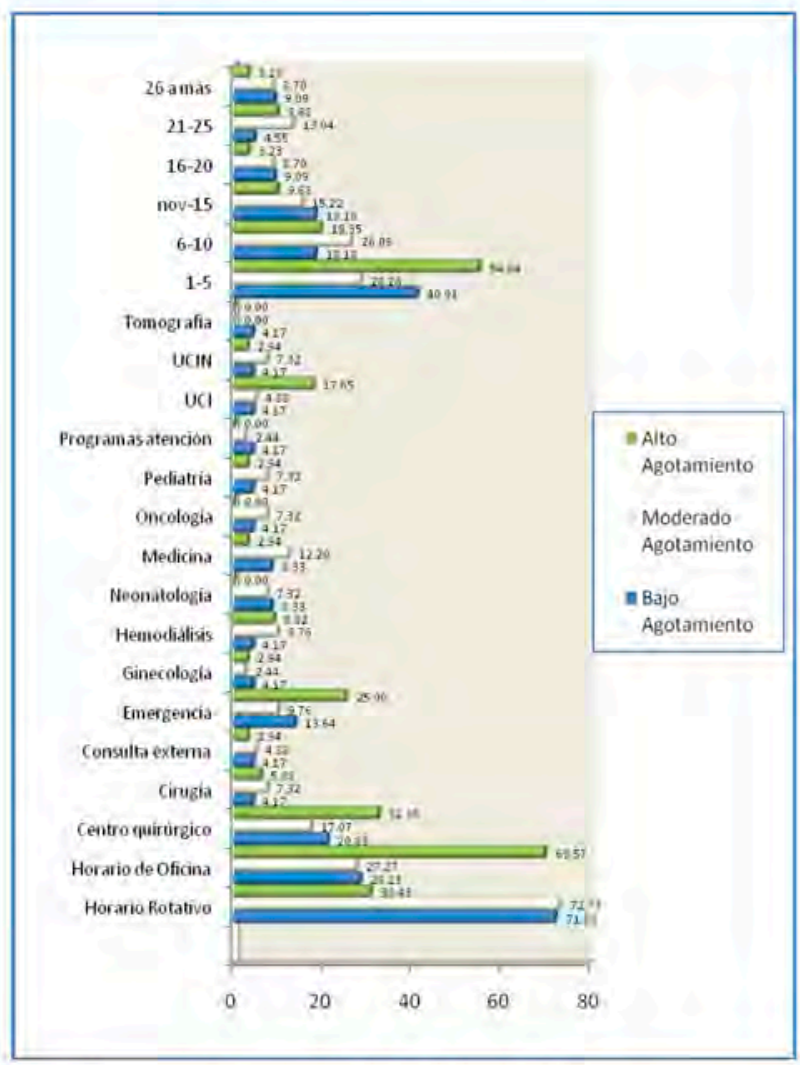

Fuente: Cuestionario sobre Estrés Laboral. Elaboración: Propia

El factor asociado al tiempo de servicio es (p: 0,468). A menor tiempo de servicio, se presenta mayor nivel de estrés. Se observa que a mayor tiempo de servicio, la frecuencia de alto agotamiento disminuye; y esta asociación es altamente significativa; puesto que se ha demostrado que el ser fijo en un trabajo aumenta el grado de satisfacción laboral.

El factor asociado al tipo de servicio es (p: 0.969). El personal de enfermería que trabaja en el Centro quirúrgico, Emergencia, y la Unidad de cuidados intensivos (UCI) presenta los mayores niveles de estrés. La diferencia es altamente significativa.

El factor asociado al horario de trabajo es (p: 0,228 ). Esto implica que las personas que trabajan en el horario de oficina tienen mayores niveles de estrés.
Gráfico $\mathbf{N}^{\circ} 07$

Distribución de frecuencia de los factores laborales según presencia de despersonalización en el personal de Enfermería del Hospital III Daniel Alcides Carrión Essalud Tacna.

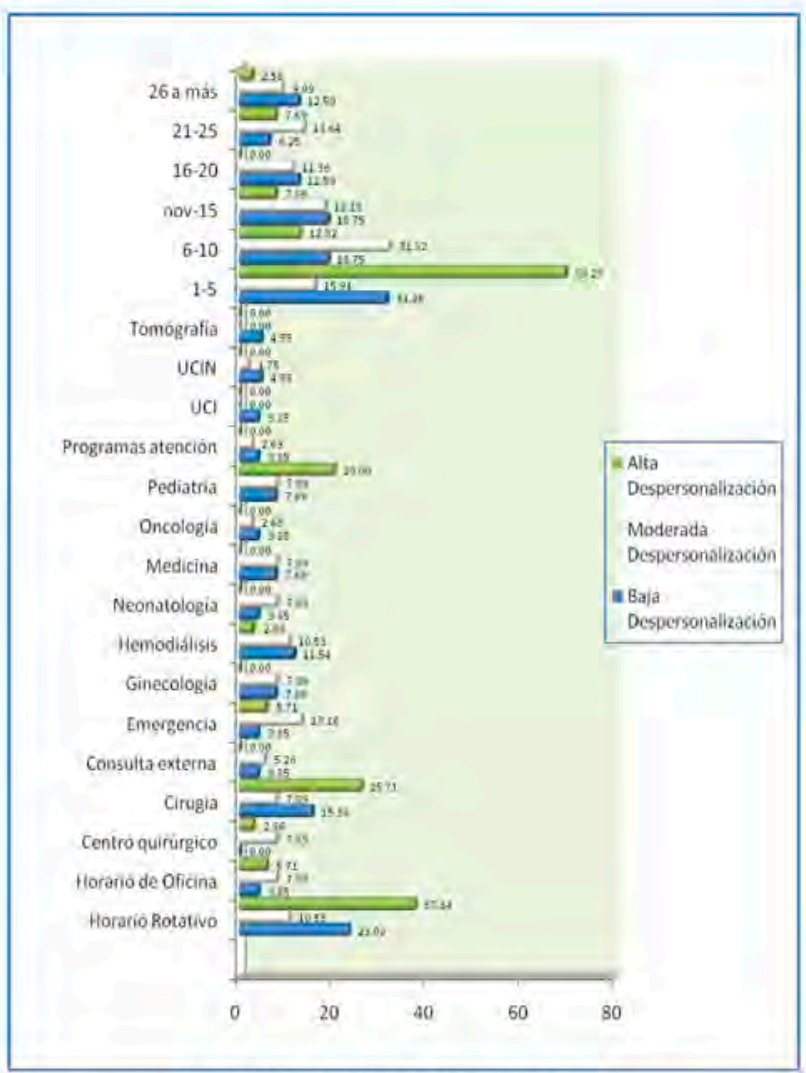

Fuente: Cuestionario sobre Estrés Laboral. Elaboración: Propia

El factor asociado al tiempo de servicio es (p: 0,189). A menor tiempo de servicio (1-5 años), se presenta mayor despersonalización. Asimismo, se observa que a mayor tiempo de servicio, la frecuencia de alto agotamiento disminuye y esta asociación es altamente significativa.

El factor asociado al tipo de servicio (p: 0.936). Destaca que el personal de enfermería que trabaja en el Centro quirúrgico, Emergencia y la Unidad de cuidados intensivos (UCI) presenta la mayor despersonalización. La diferencia es altamente significativa; no se encuentra asociación en las demás áreas de trabajo.

El factor asociado al horario de trabajo es (p: 0,234). Implica que las personas que trabajan en el horario de oficina tienen mayor despersonalización; y su asociación es altamente significativa. 
Gráfico $\mathrm{N}^{\circ} 08$

Distribución de frecuencia de los factores laborales según realización del personal de Enfermería del Hospital III Daniel Alcides Carrión Essalud Tacna.

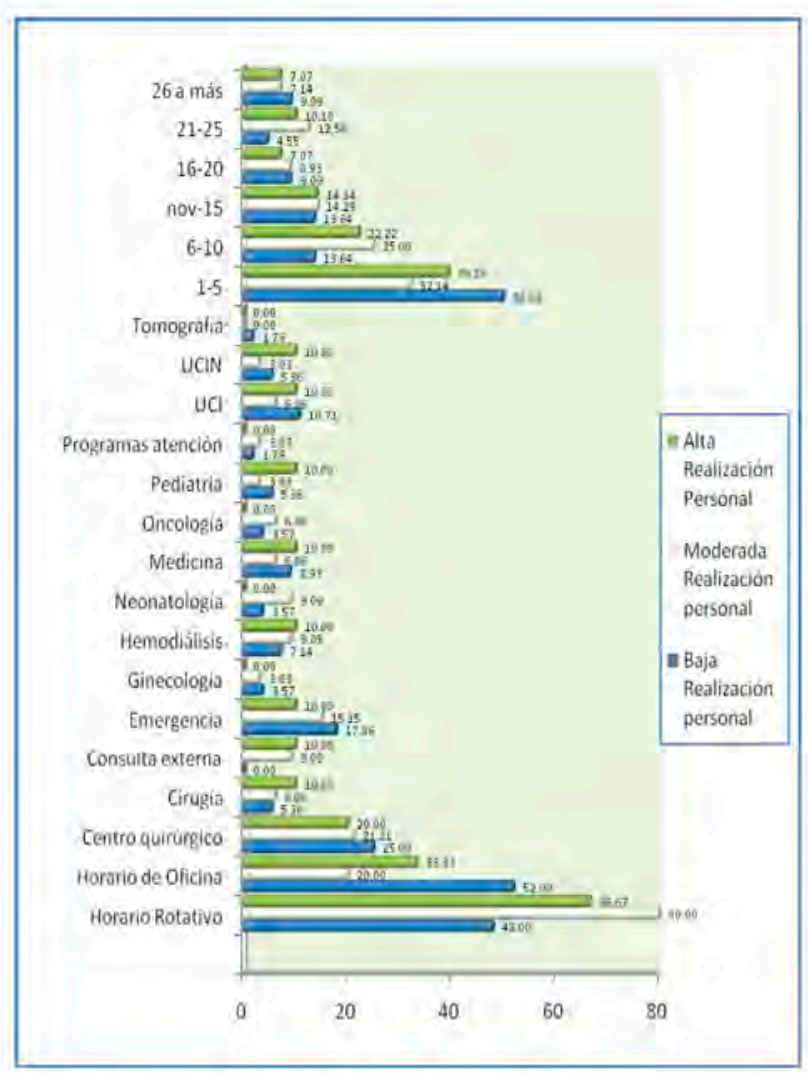

Fuente: Cuestionario sobre Estrés Laboral. Elaboración: Propia

El factor asociado al tiempo de servicio es (p: 0,839). A menor tiempo de servicio, se presenta baja realización personal. Asimismo, se observa que a mayor tiempo de servicio, la frecuencia de baja realización personal disminuye y esta asociación es altamente significativa.

El factor asociado al tipo de servicio es (p: 0.963). Se destaca que el personal de enfermería que trabaja en el Centro quirúrgico, Emergencia, y la Unidad de cuidados intensivos (UCI) presenta una baja realización personal. La diferencia es altamente significativa. Asimismo, no se encuentra asociatividad en las demás áreas de trabajo.

El factor asociado al horario de trabajo es (p: 0,459). Implica que las personas que trabajan en el horario de oficina tienen baja realización personal.

\section{DISCUSIÓN}

Los factores sociodemográficos que tienen relación con los mayores niveles de estrés (Agotamiento emocional, Despersonalización y Ausencia de realización personal) son:

- $\quad$ Estado civil; se puede observar que los solteros presentan los mayores niveles de estrés.

Edad; encontrándose que el adulto joven (31-35 años) presenta mayores niveles de estrés.

Número de hijos; esto implica que las personas que no tienen hijos, tienen los mayores niveles de estrés.

Asimismo, los factores laborales que tienen relación con los mayores niveles de estrés (agotamiento emocional, despersonalización y ausencia de realización personal) son: el tiempo de servicio, encontrándose que a menor tiempo de servicio presentan mayores niveles de estrés; el horario de trabajo, esto implica que las personas que trabajan en el horario de oficina tienen mayores niveles de estrés.

El personal de enfermeria del Hospital III Daniel Alcides Carrión ESSALUD de Tacna, que labora en los servicios criticos de Emergencia, Unidad de Cuidados Intensivos (UCI) y Sala de Operaciones (SOP), tiene mayores niveles de estrés (agotamiento emocional, despersonalización y baja realización personal); encontrándose diferencias significativas asociadas a las unidades de trabajo. Finalmente, se tiene que: el $41,41 \%$ presenta un alto agotamiento emocional; y, el $24,24 \%$ un bajo agotamiento emocional. El $37,37 \%$ presenta una alta despersonalización; y, el $28,28 \%$ una baja despersonalización. El $39,39 \%$ presenta una baja realización personal; y, el $26,26 \%$ una alta realización personal.

Se destaca que la mayoría del personal de enfermeria se encuentra con presencia del Síndrome de Burnout.

\section{REFERENCIAS}

1. ARANDA, Pando y Torres (2005) en la investigación "Factores psicosociales y síndrome de Burnout en médicos de familia. México".

2. ARÉVALO, Y. (2004). "Sobrecarga laboral y su influencia en la funcionalidad familiar de los profesionales de enfermería del Hospital Regional de Ayacucho". Ayacucho. Tesis pre grado de la Facultad de Enfermeria. Universidad Nacional de San Cristóbal de Huamanga. 
3. 3.GOMERO, R. Palomino, J. Ruiz, F. y Llap, C. (2005). El Síndrome de Burnout en personal sanitario de los hospitales de la empresa minera de Southern Perú Copper Corporation. Revista Médica Herediana. 16(4). Lima.

4. GIL MONTE, P. (2002) "Validez Factorial de la adaptación al español del Maslch Burnout InventaryGeneral Survey". Salud pública de México, vol. 44, n 1 , enero-febrero.

5. GIL MONTE, P y Peiró, JM (1997). Desgaste Psíquico en el Trabajo: El Síndrome de Quemarse. Madrid: Sintesis.

6. HARTZ, A et al. (1989) Hospital characteristics and mortality rates. The New England Journal of Medicine. Vol. 321, No. 25; p. 1720-1725.

7. HOMBRADOS, MI (1997). Estrés y Salud. Promolibro: Valencia

8. IBÁNEZ, N. y Vilaregut, A. (2004). Prevalencia del síndrome de burnout y el estado general de salud en un grupo de enfermeras de un hospital de Barcelona. Revista de Enfermería clínica. 14(3). España.

9. KEITH Davis, (2006). Comportamiento Humano en el Trabajo. Mc Graw Hill $3^{\circ}$ Edición. México. p. 557 587.

10. MANZANO GARCÍA, G. y Ramos Campos, F. (2000). Enfermería hospitalaria y síndrome de burnout. Revista de Psicología del Trabajo y de las Organizaciones, 16(2), 173-195

11. MANASSERO, M y cols. (2003) "Estrés y Burnout en la enseñanza". Palma: UIB.

12. MASLACH, CH.; Jackson, S.E. (1981a). Marlach Burnout Inventory. Manual. Palo Alto, California: Consulting Psychologists Press. 